Pacific Journal of Mathematics

ORDERED SEMIGROUPS IN PARTIALLY ORDERED 


\section{ORDERED SEMIGROUPS IN PARTIALLY ORDERED SEMIGROUPS}

\section{R. J. $\mathrm{KoCH}$}

In this note we establish a local version of the following result: a locally compact connected partially ordered non-degenerate semigroup $S$ with unit contains a non-degenerate linearly ordered local subsemigroup (containing the unit). This is an extension of a result of Gleason [2; 664] who proved a similar theorem under the additional hypotheses that

(1) $S$ is a semigroup with right invariant uniform structure and

(2) for any compact neighborhood $U$ of the identity there are nets $\left\{x_{i}\right\}$ in $S$ and $\left\{n_{i}\right\}$ integers such that $x_{i} \rightarrow e$ and $x_{i}^{n_{i}} \notin U$. A consequence of our theorem is the fact that a nondegenerate compact connected partially ordered semigroup with unit contains a standard thread joining the unit to the minimal ideal.

By a local semigroup $S$ we mean a Hausdorff space with an open subset $U$ and a multiplication $m: U \times U \rightarrow S$ which is continuous and associative insofar as is meaningful. A unit is an (unique, if it exists) element $u$ of $U$ satisfying $u x=x u=x$ for all $x \in U$. A local subsemigroup of $S$ is a subset $L$ containing the unit such that for some open set $V$ about the unit, $(V \cap L)^{2} \subset L$. We say that the local semigroup $S$ is partially ordered if the relation $\leqq$ defined by $a \leqq b$ if and only if $a=b c$ is reflexive and antisymmetric. In case $S$ is a semigroup, $S$ is partially ordered if and only if each principal right ideal has a unique generator, i.e. (assuming a unit) that $a S=b S$ implies $a=b$. In this case, $\leqq$ is also transitive.

Closure is denoted by $*$, the null set by $\square$, the boundary of $V$ by $F(V)$, and the complement of $B$ in $A$ by $A \backslash B$.

As in [4] we use the following topolopy for the space $\mathcal{S}(X)$ of nonempty closed subsets of the space $X$ : for open sets $U$ and $V$ of $X$, let $N(U, V)=\{A \mid A \in \mathscr{S}(X), A \subset U, A \cap V \neq \square\} ;$ take $\{N(U, V) \mid U, V$ open $\}$ for a sub-basis for the open sets of $\mathscr{S}(X)$. It is easy to see that if $X$ is compact Hausdorff, so is $\mathscr{S}(X)$.

THEOREM 1. Let $S$ be a locally compact partially ordered local semigroup with unit $u$, and let $U_{0}$ be a non-degenerate open connected set about $u$ with $U_{0}^{6}$ defined. Then $S$ contains a non-degenerate compact connected linearly ordered local sub-semigroup $L$ with $u \in L \subset U_{0}$.

Received December 7, 1959. This work was supported by the United States Air Force through the Air Force Office of Scientific Research, Air Research and Development Command, under Contract AF 18 (603)-89 
Proof. Let $U_{1}$ be an open set containing $u$ with $U_{1}^{*}$ compact and $U_{1}^{*} \subset U_{0}$. Define $\leqq$ on $U_{1}^{*} \times U_{1}^{*}$ by: $a \leqq b$ if and only if $a=b c$ for some $c \in U_{1}^{*}$. From the compactness of $U_{1}^{*}$ it is easily seen that Graph $(\leqq)$ is closed in $U_{1}^{*} \times U_{1}^{*}$. We show first that $\leqq$ is transitive on some neighborhood of $u$. Let $U_{2}$ be an open set about $u$ with $U_{2}^{2} \subset U_{1}$. We claim there is an open set $U$ containing $u, U \subset U_{2}$, such that if $a, b \in U^{*}$ with $a=b c$ for some $c \in U_{1}^{*}$, then $c \in U_{2}$. If this is false, then for any open set $U$ with $u \in U \subset U_{2}$, there are elements $a$ and $b$ of $U^{*}$ with $a=b c$ for some $c \in U_{1}^{*} \backslash U_{2}$. Hence there are nets $a_{a}$ and $b_{a}$ converging to $u$ with $a_{\alpha}=b_{\alpha} \cdot c_{\alpha}$ where $c_{\alpha} \in U_{1}^{*} \backslash U_{2}$. It follows that $c_{\alpha}$ must also converge to $u$, a contradiction. Since $U_{2}^{2} \subset U_{1}$ it follows that $\leqq$ is transitive on $U^{*}$. Also the restriction of $\leqq$ on $U^{*} \times U^{*}$ is closed and hence $U^{*}$ is locally convex [6]. We show next that there exists an open set $V_{1}$ with $u \in V_{1} \subset U$ such that $e^{2}=e \in V_{1}$ implies $e U_{0} e \neq e$. Suppose the contrary; we can then find a net of idempotents $e_{\alpha} \rightarrow u$ with $e_{\alpha} U_{0} e_{\alpha}=e_{\alpha}$. Let $x \in U_{0}$; then $e_{\alpha}=e_{\alpha} x e_{\alpha}$ converges to $u x u=x$, so that $x=u$ and $U_{0}$ is degenerate, a contradiction. Let $V$ be a convex open set with $u \in V \subset V^{*} \subset\left(V^{*}\right)^{2} \subset V_{1}$. Then $e^{2}=e \in V$ implies $e U_{0} e \neq e$.

Let $\mathscr{C}$ denote the collection of all closed chains $C$ in $U^{*}$ with $u \in C$, $C \cap S \backslash V \neq \square$, and $(C \cap V)^{2} \subset C$. Note that $\mathscr{C} \neq \square$, for if $a \in F(V)$, then the elements $u$ and $a$ constitute an element of $\mathscr{C}$.

(i) $\mathscr{C}$ is closed in $\mathscr{P}\left(U^{*}\right)$. We will show that $\mathscr{C}$ is an intersection of closed set. Since the collection of all closed chains which contain $u$ and meet $S \backslash V$ is closed [4], it remains to show that the collection of closed chains $C$ satisfying $(C \cap V)^{2} \subset C$ is closed. Suppose $A$ is a closed chain with $(A \cap V)^{2} \not \subset A$; then there are elements $a$ and $b$ of $A \cap V$ with $a b \in S \backslash A$. Hence there exist open sets $U_{a}, U_{b}$, and $W$ containing $a, b$, and $A$ respectively, with $U_{a} \cdot U_{b} \cap W=\square$. Now $N\left(W, U_{a}\right) \cap$ $N\left(W, U_{b}\right)$ is an open set about $A$, and contains no chain $C$ with $(C \cap$ $V)^{2} \subset C$. This establishes (i).

As in [4], we define $L(x)=\{y \mid y \leqq x\}, M(x)=\{y \mid x \leqq y\}$, and $(x, y)=$ $\{z \mid x<z<y\}$. Let $\delta$ be an open cover of $U^{*}$, and define a subset $M_{\delta}$ of $\mathscr{S}\left(U^{*}\right)$ by: $C \in M_{\delta}$ if and only if $C$ is a closed chain in $U^{*}$, and for any $x$ and $y$ in $C$ with $x<y$ and $(x, y) \cap C=\square$, there exists $D \in \delta$ such that $D^{*}$ meets both $L(x) \cap C$.

(ii) $M_{\delta} \cap \mathscr{C} \neq \square$ for any open cover $\delta$ of $U^{*}$. Let $\delta$ be an open cover of $U^{*}$, and let $\mathscr{D}$ be the collection of all closed chains $C$ with $u \in C \subset U, C \in M_{\delta}$, and $(V \cap C)^{2} \subset C$. Let $\tau$ be a maximal tower in $\mathscr{D}$, and let $T=U \tau$. Then $T^{*}$ is a closed chain, $u \in T^{*} \subset U^{*}$, and $\left(V \cap T^{*}\right)^{2} \subset$ $T^{*}$. As in [4], $T^{*} \in M_{\delta}$, and it remains to show that $T^{*} \in \mathscr{C}$, i.e., that $T^{*} \cap S \backslash V \neq \square$. Suppose $T^{*} \subset V$; (note then that $T=T^{*}$ ) then since $(T \cap V)^{2} \subset T, T$ is a compact chain and a semigroup. Let $e=\inf T$. Since $e^{2} \leqq e$ and $e^{2} \in T$ we have $e^{2}=e$. We show next that $e$ is a zero 
for $T$. Let $y \in T$, then $e y \in T$ and $e y \leqq e$, so $e y=e$ and $e$ is a left zero for $T$. Hence the minimal ideal $K$ of $T$ consists of left zeros for $T$ [1]. Let $f \in K$; then $e \leqq f$ so there exists $c \in U_{1}^{*}$ with $e=f c$. Therefore $f=f e=e$, and $e$ is the unique left zero, and hence a zero for $T$. Let $W \in \delta$ with $e \in W$. If $e U_{0} e \cap W \cap V$ contains an idempotent $g \neq e$, then $T \cup g$ is a semigroup: for if $x \in T$ then $x g=x(e g)=e g=g$ and $g x=(g e) x=g(e x)=g e=g$. Also $T \cup g$ is a chain, so by the maximality of $\tau, T=T \cup g$, a contradiction.

Hence we may assume that $e U_{0} e \cap W \cap V$ has a unique idempotent $e$. Since $\leqq$ is antisymmetric, the maximal subgroup of $S$ containing $e$ is $e$. Also $e U_{0} e$ is a local semigroup with unit $e, e U_{0} e \neq e$, and $e$ is not isolated in $e U_{0} e$ which is the continuous image of $U_{0}$ and hence connected. Hence $[5 ; 122]$ there is a non-degenerate one parameter local semigroup $A$ with $e \in A \subset e U_{0} e \cap W \cap V$; let $a \in A$ with $a \neq e$ and $a^{2} \in A$. Define $a^{0}=e$ and let $B_{k}=\bigcup_{n=0}^{k} a^{n}[a, e], B_{\infty}=\bigcup_{n=0}^{\infty} a^{n}[a, e]$ where $[a, e]$ denotes the sub-arc of $A$ from $a$ to $e$. We assume temporarily that all products involved in forming $B_{k}$ and $B_{\infty}$ are defined. Each of the sets $a^{n}[a, e]$ is a compact connected chain (hence an arc) with minimal element $a^{n+1}$ and maximal element $a^{n}$. Hence $B_{k}$ is a compact connected chain from $a^{k+1}$ to $e$. Also $B_{\infty}$ is a connected chain, hence $B_{\infty}^{*}$ is a closed connected chain. Using the easily established commutativity of $B_{k}$ and $B_{\infty}^{*}$ it follows that for $x \in T$ and $b \in B_{k}$ (or $B_{\infty}^{*}$ ) then $x b=x(e b)=(x e) b=$ $e b=b$, and similarly $b x=b$. Hence $\left[\left(T \cup B_{k}^{2}\right) \cap V\right]^{2} \subset T \cup\left(B_{k}^{2} \cap V\right)^{2}$ and similarly with $B_{k}$ replaced by $B_{\infty}^{*}$. We distinguish two cases:

Case 1: For some $k \geqq 0, a^{k+1} \in V$ and $a^{k+2} \notin V$. Then since $V$ is convex, $a^{0}, a, \cdots, a^{k+1}$ are in $V$ and all products involved in forming $B_{k}$ are defined, so that $B_{k} \subset V$ and $B_{k+1} \not \subset V$. We show first that $B_{k}^{2} \cap V \subset$ $B_{k}$. Let $z \in B_{k}^{2} \cap V$; then $z=x y$ with $x, y \in B_{k}$, so $x=a^{n} x^{\prime}$ and $y=a^{m} y^{\prime}$ with $x^{\prime}$ and $y^{\prime}$ in $[a, e]$. Hence $x y=a^{m+n} x^{\prime} y^{\prime}$. If $x^{\prime} y^{\prime} \in A$, then since $z \in V$ it follows that $m+n \leqq k$. If $x^{\prime} y^{\prime} \notin A$, then $x^{\prime} y^{\prime}=a t$ for some $t \in A$, so $x y=a^{m+n+1} t$ and $m+n+1 \leqq k$. In either case, then, $z \in B_{k}$. Note that $\left(T \cup B_{k}\right)^{2} \in M_{\delta}$ since $B_{k}^{2}$ is a connected chain. Also [ $\left(T \cup B_{k}^{2}\right) \cap$ $V]^{2} \subset T \cup\left(B_{k}^{2} \cap V\right)^{2} \subset T \cup B_{k}^{2}$, so that $T \cup B_{k}^{2} \in \mathscr{D}$. This contradicts the maximality of $\tau$.

Case 2: $a^{k} \in V$ for each $k \geqq 0$. Using the convexity of $V$ we see that all products involved in forming $B_{\infty}$ are defined, and $B_{\infty}=B_{\infty}^{2} \subset V$, hence $B_{\infty}^{*}=B_{\infty}^{* 2}$. Since $B_{\infty}^{*}$ is a connected chain, it follows that $T \cup$ $B_{\infty}^{*} \in M_{\delta}$. Also $\left[\left(T \cup B_{\infty}^{*}\right) \cap V\right]^{2} \subset T \cup B_{\infty}^{*}$, so that $T \cup B_{\infty}^{*} \in \mathscr{D}$, a contradiction to the maximality of $\tau$. The proof of (ii) is now complete.

(iii) $M_{\delta} \cap \mathscr{C}$ is closed for each finite open cover $\delta$ of $U^{*}$.

This proof is similar to that in [4], and is omitted.

For any finite open cover $\delta$ of $U^{*}$, let $P_{\delta}=M_{\delta} \cap \mathscr{C}$. The collection of sets $\left\{P_{\delta}\right\}$ is a descending family, so $\cap P_{\delta} \neq \square$. If $C \in \bigcap P_{\delta}$, 
then as shown in [4], $C$ is an arc. Clearly $C$ is a local semigroup, and the proof is complete.

In what follows, a standard thread is a compact connected semigroup irreducibly connected between a zero and a unit. The structure of standard threads is known [5; 130]. The example in [4] shows that a compact connected semigroup with zero and unit need not contain a standard thread joining the zero to the unit. The problem of finding standard threads joining zero to unit has an affirmative solution in case either

(1) $S$ is compact, connected, and one-dimensional [3], or

(2) $S$ is compact, connected, and each element is idempotent [4]. A third solution is given by the following corollary.

COROLlaRY 1. If $S$ is a non-degenerate compact connected partially ordered semigroup with unit $u$, then the minimal ideal $K$ consists of left zeros for $S, K$ consists of the set of minimal elements, and some elements of $K$ can be joined by a standard thread to the unit.

Proof. Note that Graph ( $\leqq$ ) is closed since $S$ is compact. Let $G$ be a compact group in $S$, with unit $e$. Since $x^{2} \leqq x$ for each $x \in S$, then for $x \in G$ we have $e \geqq x \geqq x^{2} \geqq \cdots$, and $\left\{x^{n}\right\}$ clusters at an idempotent, which must be $e$. We conclude that $x=e$, and hence that each compact group in $S$ is trivial. From this fact it is clear that $K$ is proper, for otherwise $K=S$ would be a compact group [1]. From the fact that $a S=b S$ implies $a=b$ we conclude that each minimal right ideal is a single element, hence each element of $K$ is a left zero for $S$ [1]. Since a minimal element $x$ of $S$ is characterized by the equality $x S=x$, it is clear that $K$ consists of the set of minimal elements of $S$, and hence that $S \backslash K$ is convex. In the proof of the Theorem, we take $S=U_{0}=U_{1}=U_{2}=U$, and $V=S \backslash K$. Hence there is a compact connected linearly ordered local semigroup $L$ containing $u$, with $L \cap S \backslash V \neq \square$. Since the elements of $K$ are minimal it follows that $L$ is a semigroup, and hence a standard thread.

\section{REFERENCES}

1. A. H. Clifford, Semigroups containing minimal ideals, Amer. J. Math. 70 (1948), 521-526.

2. A. M. Gleason, Arcs in locally compact groups, Proc. Nat. Acad. Science 36 (1950), 663-667.

3. R. P. Hunter, On the semigroup structure of continua, Trans. Amer. Math. Society. 93 (1959), 356-368.

4. R. J. Koch, Arcs in partially ordered spaces, Pacific J. Math. 9 (1959), 723-728.

5. P. S. Mostert and A. L. Shields, On the structure of semigroups on a compact manifold with boundary, Amer. Math 65 (1957), 117-143.

6. L. Nachbin, Sur les spaces topologiques ordonnés C. R. Acad. Sci. Paris 226 (1948), 


\section{PACIFIC JOURNAL OF MATHEMATICS}

\section{EDITORS}

David GILbarg

Stanford University

Stanford, California

\section{F. H. BRowneLL}

University of Washington

Seattle 5, Washington

\section{A. L. Whiteman}

University of Southern California Los Angeles 7, California

\section{J. PAIGe}

University of California

Los Angeles 24, California

\section{ASSOCIATE EDITORS}
E. F. BECKENBACH
T. M. CHERRY
D. DERRY

\author{
E. HEWITT \\ A. HORN \\ L. NACHBIN
}

\author{
M. OHTSUKA \\ H. L. ROYDEN \\ M. M. SCHIFFER
}

E. SPANIER

E. G. STRAUS

F. WOLF

\section{SUPPORTING INSTITUTIONS}

\author{
UNIVERSITY OF BRITISH COLUMBIA \\ CALIFORNIA INSTITUTE OF TECHNOLOGY \\ UNIVERSITY OF CALIFORNIA \\ MONTANA STATE UNIVERSITY \\ UNIVERSITY OF NEVADA \\ NEW MEXICO STATE UNIVERSITY \\ OREGON STATE COLLEGE \\ UNIVERSITY OF OREGON \\ OSAKA UNIVERSITY \\ UNIVERSITY OF SOUTHERN CALIFORNIA
}

\author{
STANFORD UNIVERSITY \\ UNIVERSITY OF TOKYO \\ UNIVERSITY OF UTAH \\ WASHINGTON STATE COLLEGE \\ UNIVERSITY OF WASHINGTON \\ AMERICAN MATHEMATICAL SOCIETY \\ CALIFORNIA RESEARCH CORPORATION \\ HUGHES AIRCRAFT COMPANY \\ SPACE TECHNOLOGY LABORATORIES \\ NAVAL ORDNANCE TEST STATION
}

\footnotetext{
Mathematical papers intended for publication in the Pacific Journal of Mathematics should be typewritten (double spaced), and the author should keep a complete copy. Manuscripts may be sent to any one of the four editors. All other communications to the editors should be addressed to the managing editor, L. J. Paige at the University of California, Los Angeles 24, California.

50 reprints per author of each article are furnished free of charge; additional copies may be obtained at cost in multiples of 50 .
}

The Pacific Journal of Mathematics is published quarterly, in March, June, September, and December. The price per volume (4 numbers) is $\$ 12.00$; single issues, $\$ 3.50$. Back numbers are available. Special price to individual faculty members of supporting institutions and to individual members of the American Mathematical Society: $\$ 4.00$ per volume; single issues, $\$ 1.25$.

Subscriptions, orders for back numbers, and changes of address should be sent to Pacific Journal of Mathematics, 2120 Oxford Street, Berkeley 4, California.

Printed at Kokusai Bunken Insatsusha (International Academic Printing Co., Ltd.), No. 6, 2-chome, Fujimi-cho, Chiyoda-ku, Tokyo, Japan.

PUBLISHED BY PACIFIC JOURNAL OF MATHEMATICS, A NON-PROFIT CORPORATION

The Supporting Institutions listed above contribute to the cost of publication of this Journal, but they are not owners or publishers and have no responsibility for its content or policies. 


\section{Pacific Journal of Mathematics}

\section{Vol. 10, No. $4 \quad$ December, 1960}

M. Altman, An optimum cubically convergent iterative method of inverting a linear bounded operator in Hilbert space . . . . . . . . . . . . . . . . . . . . . . . . . . 1107

Nesmith Cornett Ankeny, Criterion for rth power residuacity ................. 1115

Julius Rubin Blum and David Lee Hanson, On invariant probability measures I . . . . . 1125

Frank Featherstone Bonsall, Positive operators compact in an auxiliary topology ..... 1131

Billy Joe Boyer, Summability of derived conjugate series . . . . . . . . . . . . . . . . 1139

Delmar L. Boyer, A note on a problem of Fuchs . . . . . . . . . . . . . . . . . 1147

Hans-Joachim Bremermann, The envelopes of holomorphy of tube domains in infinite

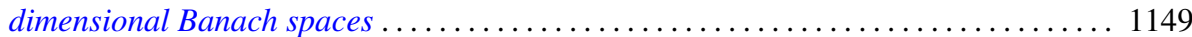

Andrew Michael Bruckner, Minimal superadditive extensions of superadditive

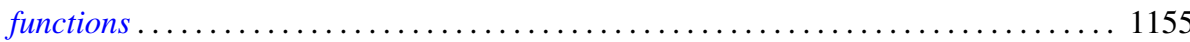

Billy Finney Bryant, On expansive homeomorphisms .................... 1163

Jean W. Butler, On complete and independent sets of operations in finite algebras . . . . . 1169

Lucien Le Cam, An approximation theorem for the Poisson binomial distribution ...... 1181

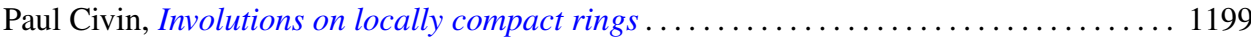

Earl A. Coddington, Normal extensions of formally normal operators . . . . . . . . . 1203

Jacob Feldman, Some classes of equivalent Gaussian processes on an interval ........ 1211

Shaul Foguel, Weak and strong convergence for Markov processes . . . . . . . . . . . 1221

Martin Fox, Some zero sum two-person games with moves in the unit interval ........ 1235

Robert Pertsch Gilbert, Singularities of three-dimensional harmonic functions . . . . . . . 1243

Branko Grünbaum, Partitions of mass-distributions and of convex bodies by

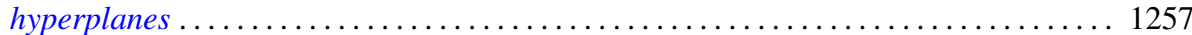

Sidney Morris Harmon, Regular covering surfaces of Riemann surfaces ........... 1263

Edwin Hewitt and Herbert S. Zuckerman, The multiplicative semigroup of integers

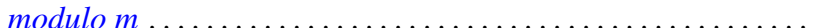

Paul Daniel Hill, Relation of a direct limit group to associated vector groups . ......... 1309

Calvin Virgil Holmes, Commutator groups of monomial groups . .

James Fredrik Jakobsen and W. R. Utz, The non-existence of expansive homeomorphisms

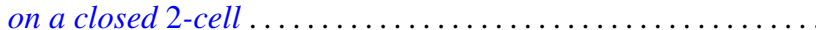

John William Jewett, Multiplication on classes of pseudo-analytic functions . . . . . . . 1323

Helmut Klingen, Analytic automorphisms of bounded symmetric complex domains . . . . 1327

Robert Jacob Koch, Ordered semigroups in partially ordered semigroups . . . . . . . . 1333

Marvin David Marcus and N. A. Khan, On a commutator result of Taussky and

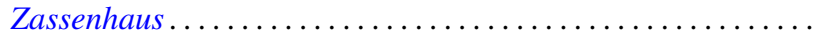

John Glen Marica and Steve Jerome Bryant, Unary algebras......

Edward Peter Merkes and W. T. Scott, On univalence of a continued fraction . . . . . . . 1361

Shu-Teh Chen Moy, Asymptotic properties of derivatives of stationary measures . . . . . 1371

John William Neuberger, Concerning boundary value problems . . . . . . . . . . . 1385

Edward C. Posner, Integral closure of differential rings . . . . . . . . . . . . . . . . . 1393

Marian Reichaw-Reichbach, Some theorems on mappings onto . . . . . . . . . . . . . 1397

Marvin Rosenblum and Harold Widom, Two extremal problems . . . . . . . . . . . . . . . . 1409

Morton Lincoln Slater and Herbert S. Wilf, A class of linear differential-difference

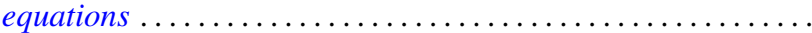

Charles Robson Storey, Jr., The structure of threads . . . . . . . . . . . . . . . . . . 1429

J. François Treves, An estimate for differential polynomials in $\partial / \partial z_{1},, \cdots, \partial / \partial z_{-} n \ldots \ldots 1447$

J. D. Weston, On the representation of operators by convolutions integrals . . . . . . . . 1453

James Victor Whittaker, Normal subgroups of some homeomorphism groups ......... 1469 